\title{
Erratum: Erratum: On critical exponents for self-similar collapse
}

\author{
Riccardo Antonelli and Ehsan Hatefi \\ Scuola Normale Superiore and I.N.F.N., \\ Piazza dei Cavalieri 7, Pisa 56126, Italy \\ E-mail: riccardo.antonelli@sns.it, ehsan.hatefi@sns.it
}

ERRATUM TO: JHEP10(2020)104

ARXIV EPRINT: 1912.06103

Author Ehsan Hatefi has just this affiliation:

Scuola Normale Superiore and I.N.F.N., Piazza dei Cavalieri 7, 56126, Pisa, Italy

Open Access. This article is distributed under the terms of the Creative Commons Attribution License (CC-BY 4.0), which permits any use, distribution and reproduction in any medium, provided the original author(s) and source are credited. 